\title{
DIMENSIONES DE ANÁLISIS DEL DIAGNÓSTICO EN EDUCACIÓN: EL DIAGNÓSTICO DEL CONTEXTO FA- MILIAR
}

\section{[Analysis dimensions in educational diagnosis: Diagnosis of Family Context]}

por

$\underline{\text { Article record }}$

About authors

$\underline{\text { HTML format }}$

\author{
Esperanza Ceballos \\ (eceballo@ull.es)
}

Ficha del artículo

Sobre los autores

Formato HTML

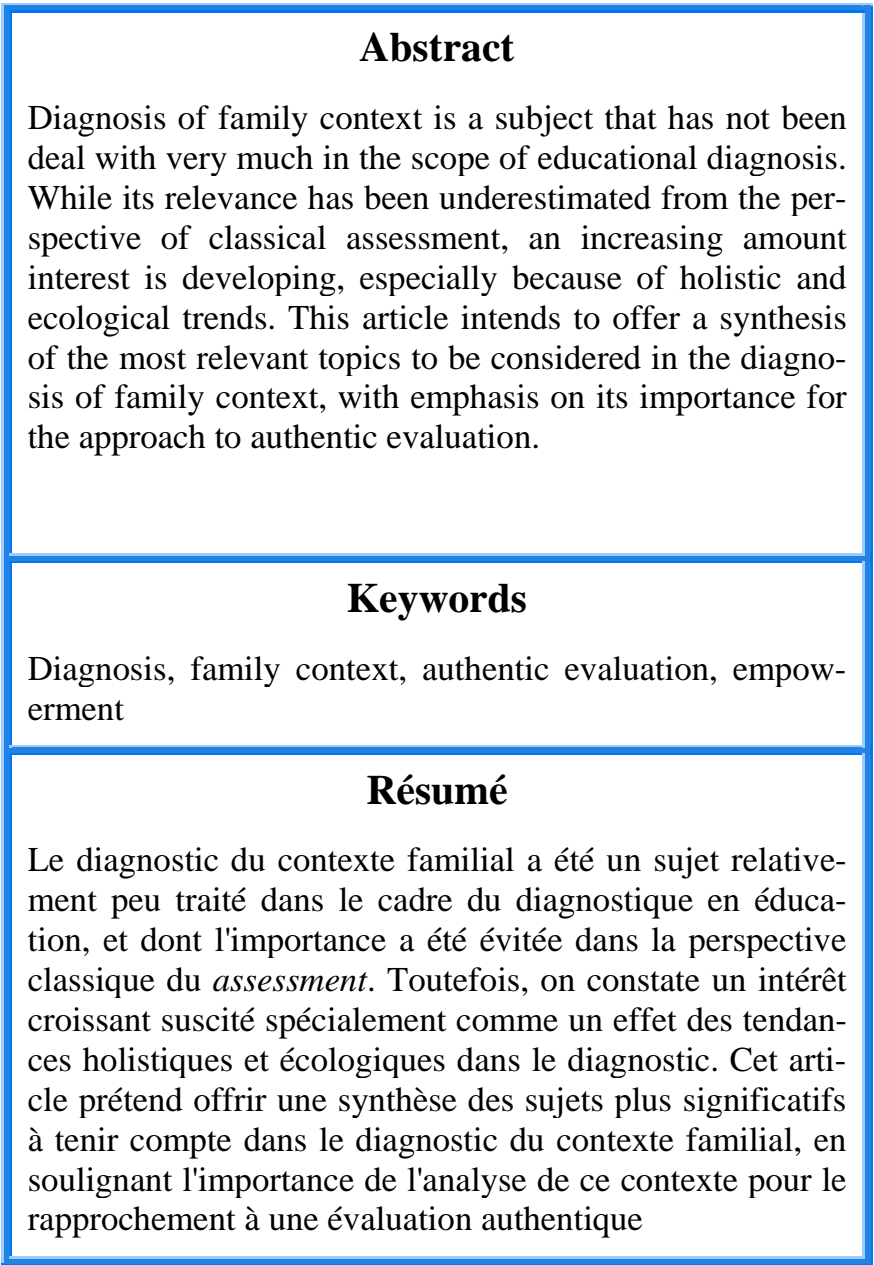

\section{Resumen}

El diagnóstico del contexto familiar ha sido un tema relativamente poco tratado en el ámbito del diagnóstico en educación, y cuya relevancia ha sido soslayada desde la perspectiva clásica del assessment. Sin embargo, se constata un interés creciente suscitado especialmente al hilo de las tendencias holísticas y ecológicas en el diagnóstico. Este artículo pretende ofrecer una síntesis de los tópicos más relevantes a tener en cuenta en el diagnóstico del contexto familiar, subrayando la importancia del análisis de este contexto para la aproximación a una evaluación auténtica.

\section{Descriptores}

Diagnóstico, contexto familiar, evaluación auténtica, fortalecimiento.

\section{Mots Clé}

Diagnostique, contexte familial, évaluation authentique, empowerment 


\section{Introducción: La importancia de la familia y de la educación para la vida familiar}

La familia es el núcleo esencial de desarrollo humano. Es su primera y más básica fuente de socialización; en su seno se construye el apego, base de la autoestima, y el autoconcepto para la vida adulta. Y aún más, es el escenario que nos permite aprender desde niños a afrontar retos y a asumir responsabilidades (Palacios y Rodrigo, 1998). En la familia se imparte un currículum «para la vida», pozo inagotable de conocimientos imprescindibles en el reto vital. Por tanto, no es de extrañar que, para la mayoría de las personas, la familia constituya el aspecto más importante de su vida, del cual depende básicamente su felicidad, sin parangón con los logros escolares, profesionales, económicos... Sin embargo, es cierto que mientras realizamos una considerable inversión educativa para conseguir éxito académico o profesional, al parecer nos «preparamos» menos concienzudamente en lo que se refiere a nuestra vida familiar cotidiana, y a las decisiones que tomamos respecto a ella. $\mathrm{O}$ mejor dicho, aunque la educación familiar siempre haya existido como fenómeno de transmisión social, dicho aprendizaje no se ha revestido de un soporte formal tan explícito. Pues tradicionalmente la comunidad, y el propio círculo familiar de referencia, se encargaban de transmitir los conocimientos y habilidades necesarios para desarrollar una vida familiar «adecuada» y acorde a los valores sociales imperantes.

Aunque la idea de «recibir una educación para la vida familiar» fuera de estos parámetros resulte todavía novedosa, hay que decir que cada vez esta opción es considerada menos extraña. De hecho, en las sociedades cultural y económicamente desarrolladas, son cada vez más habituales las demandas de formación para educar correctamente a los hijos (Martínez González, 1999), y en general, para alcanzar una vida familiar más plena para todos sus componentes. Estas demandas de formación y asesoramiento probablemente tienen que ver con el debilitamiento de los pilares tradicionales de la educación fami$\operatorname{liar}^{[i]}$. Las familias viven cada vez físicamente más alejadas (la movilidad laboral obliga a las familias a distanciarse, las mejoras en el nivel de vida permiten vivir en residencias independientes, y existe una cierta infravaloración de la «sabiduría de nuestros mayores»...), el influjo de la comunidad como núcleo de aprendizaje y control social se diluye en la vida urbana, y el poder de la religión para controlar las conductas se desvanece de forma progresiva en las sociedades desarrolladas y crecientemente más laicas.

Mientras tanto desde la escuela, los servicios sociales, el sector sanitario... y en general desde la sociedad, se responsabiliza a las familias de las consecuencias en sus hijos (ya sea por sus resultados académicos, por sus conductas sociales, por sus éxitos laborales...); es decir, los problemas de la juventud actual se achacan, en gran parte y sobre todo, a la deficiente labor educativa de las familias. Familias, que han perdido sus núcleos tradicionales de apoyo y se sienten desconcertadas ante la variedad de demandas que exige la vida familiar, y las dificultades para conducirse de forma apropiada en la realización de sus distintas tareas: la crianza de los hijos, la resolución de los conflictos entre los miembros de la familia, la conciliación de la vida laboral y familiar, la gestión económica y doméstica...

En definitiva, se plantea la necesidad de una «educación familiar» como medio de apoyo a las familias, tanto para ayudarlas a hacer frente a sus dificultades, como, para simplemente, contribuir a su mejora y al aumento de su satisfacción... Por ello, empieza a ser normal que las familias acudan a servicios de orientación educativa, que funcionan como recursos externos para fortalecer a las familias y para ayudarlas a conocerse mejor a sí mismas. Estos recursos serán más crucia- 
les en cuanto la familia disponga de menos recursos internos (bajo nivel de cohesión familiar), y se encuentre sometida a un mayor nivel de estrés (Simón, Correa, Rodrigo y Rodríguez, 1998). No obstante, debemos señalar que la orientación familiar es el ámbito menos desarrollado de la orientación educativa (Álvarez González, 2003; Santana, 1993), al menos en España, si bien en los últimos tiempos se advierte un gran dinamismo plasmado en un aumento de las publicaciones, y en la celebración de congresos alusivos a la educación familiar.

\section{La familia en la sociedad postmo- derna: el diagnóstico macrosistémico}

En la sociedad postmoderna el cambio es un síndrome permanente (Santana, 2001). Todo es inestable: las ideologías, los trabajos, los afectos... y por supuesto, la familia no se sustrae a ese destino. Los cambios en la familia, y hasta en el propio concepto de familia, están a la orden del día (Cusinato, 2002). Las familias de hoy son «inestables» $\mathrm{y}$ tienden a la ruptura (constatada en el aumento de los divorcios); sin embargo la posibilidad de contraer nuevas nupcias también ha aumentado. Este aumento en el número relativo de parejas (casadas o no) no va, sin embargo, acompasado por un incremento de la natalidad. En la sociedad del bienestar los hijos son un bien escaso, ya que suponen un elevado coste económico y personal. Así, afirman Pérez Díaz, Chuliá y Valiente (2000) que en la incipiente sociedad del bienestar española la respuesta de las familias ha sido «reducir hijos» y «ampliar patrimonio», en contraste con el empobrecimiento relativo de los hogares con varios hijos. Por eso la pauta habitual es que nuestras familias tiendan a ser más «largas y estrechas». Más estrechas porque se tienen menos hijos, que resultan en todo caso de una elección consciente y deseada (Giddens, 1999), y en general, conviven menos miembros en el mismo hogar ${ }^{[i i]}$, aunque en España los hijos tienden a permanecer en el hogar familiar hasta edades más avanzadas. Y también son familias más lar- gas porque presentan una mayor distancia intergeneracional, principalmente debida al aumento de la edad media para tener al primer hijo, y a la mayor esperanza de vida, que permite convivir por más tiempo, por ejemplo, a abuelos y nietos.

En realidad estos cambios se habían avisado desde los años sesenta, aunque podemos encontrar su raíz en la revolución industrial y la incorporación de la mujer al mundo laboral. Sin embargo, ha sido el peso del feminismo el que ha venido replanteando los roles de la mujer y el hombre en la familia, cuestionando los valores tradicionales, y contribuyendo a «equilibrar», al menos hasta cierto punto, la balanza del poder en el seno de los hogares ${ }^{[i i i]}$. Si la sociedad reclamaba la necesidad de un orden social y político de carácter democrático, estos valores se transferían también a la familia, imponiendo una suerte de «democracia familiar» como estándar educativo y relacional entre sus miembros. Incluso, la extensión de los valores democráticos le han valido la denominación de padres democráticos, a aquellos que negocian y toman decisiones conjuntamente con sus hijos, en contraposición a los padres autoritarios y a los permisivos, de acuerdo con la clásica tipología de Baumrind (1973). Las consecuencias de la aplicación de esta metáfora no han sido siempre bien acogidas, resaltándose su inadecuación, especialmente en el caso de las relaciones padres-hijos, dada la natural asimetría de poder y razonamiento, en los primeros años de edad. Y el peligro de caer en el excesivo permisivismo (laissez faire), que resalta Esteve (2003), al referirse al modelo de «educación como libre desarrollo», cuyo exponente más significativo encuentra en el famoso libro del Dr. Spock «Baby and child care» ${ }^{[\mathrm{iv}]}$.

Como venía diciendo, otro aspecto a destacar es la pluralidad de las fórmulas familiares, que nos lleva a hablar de una diversidad en el concepto de familia, y una creciente tolerancia social respecto a estas familias «diferentes». Quizás la más evidente sea la 
aceptación de las parejas no casadas con hijos, que por ejemplo, en la UE alcanzan como promedio un $20 \%$, en el Reino Unido un $30 \%$, y en Dinamarca llegan a un $40 \%$ (Eurostat, 1998). También es notorio el crecimiento (explícito) de las familias monoparentales, constituidas principalmente por la madre y su/s hijo/s; que en nuestro país ya suponen el $7 \%$ de las familias, una cifra que parece ir en progreso, pues han aumentado un $20 \%$ en los últimos tres años, de acuerdo con los datos del Instituto Nacional de Estadística recogidos por el diario El País (2003). Otros modelos familiares en alza son las familias adoptivas, reconstituidas, parejas sin hijos... entre otras, y con menor grado de aceptación, las parejas homosexuales (con o sin hijos). En todo caso, el ingrediente principal para definir el conjunto familiar es el mantenimiento de lazos de afecto y la existencia de solidaridad mutua entre los miembros (Alberdi, 1999), con independencia de los vínculos de sangre, y de la convivencia bajo un mismo techo (por ejemplo, gana adeptos la fórmula de pareja LAT o living apart together).

\section{El Diagnóstico del contexto familiar}

A tenor de lo expuesto, se evidencia la complejidad y versatilidad de los sistemas familiares, y al mismo tiempo se deja constancia de que el contexto familiar no puede ser obviado en el análisis de las realidades humanas. Es más, en un diagnóstico de naturaleza holística la presencia de la familia es reconocida como un elemento de enorme relevancia durante todo el ciclo vital, que es apropiadamente subrayado por el enfoque ecológico-sistémico. Esto no equivale a decir que el papel de la familia sea exclusivo, sino que más bien la clave se encuentra en las interrelaciones entre los individuos, el sistema familiar, y el resto de sistemas de influencia (Hernández Blasi, 1996). Aunque estas relaciones se establecen en todas direcciones, y de forma dinámica, también es cierto que la familia juega un papel central en la conformación de los individuos, que a su vez, redefinen el funcionamiento de instituciones educativas, empresas... y hasta gobiernos.

En contraste, la perspectiva clásica del assessment, más centrada en el diagnóstico individual del sujeto, ha soslayado a la familia relativamente, sacándola a colación, sobre todo, como fuente de información acerca del sujeto. Sin embargo, la consideración ecológica de los contextos de desarrollo, resitúa a la familia como contexto básico en el diagnóstico. Al mismo tiempo, el carácter participativo del diagnóstico, otorga a la familia un papel activo en la construcción del diagnóstico, ¿pero es necesario el análisis del contexto familiar en todos los diagnósticos educativos?

Justamente, una de las competencias a desarrollar por el experto en diagnóstico es la de seleccionar los elementos de análisis más adecuados en cada caso. En principio, entiendo que el diagnóstico del contexto familiar debe estar patente cuando se presuma la relevancia de dicho contexto como una de las causas que determinan la situación objeto de análisis. O bien, el diagnóstico familiar puede resultar de interés en sí mismo como contexto educativo, puesto que la familia es el núcleo de socialización por excelencia, y presenta sus propias e ineludibles necesidades educativas. Sin olvidar que la familia es el escenario en donde se imparte la «educación para la vida», asignatura en la que la familia y la escuela, entre otros, ejercerán una decisiva influencia ${ }^{[\mathrm{v}]}$.

\subsection{Hacia la participación y el «forta- lecimiento» de las familias.}

Si hablamos de las funciones del diagnóstico del contexto familiar señalaré que, en coherencia con los principios del Diagnóstico en Educación, se dirigirán a la corrección cuando sea necesario, pero también, y sobre todo, a la prevención y al desarrollo de las familias. La finalidad última será colaborar para que las familias aprendan a conocerse mejor a sí mismas, comprendan mejor sus 
mecanismos de funcionamiento, y encuentren sus propios caminos para una mayor realización personal y familiar, lo cual conecta directamente con la perspectiva del «fortalecimiento». Desde la orientación familiar, Ausín, López y Martín-Izard (1998) señalan esta última, o de empowerment, como la primera línea a destacar; seguida de la línea de prevención, en la que el objetivo no es lograr una «familia fuerte», sino una «familia saludable» (si bien encuentran en ambas líneas intensas interrelaciones); y por último, señalan la de modificación, para familias con elevadas necesidades (grandes discrepancias entre la situación actual y deseable).

Otra cuestión, muy relacionada con lo anterior es el principio de la participación de la familia en la construcción del diagnóstico y de las alternativas para la toma de decisiones. En este sentido recordaré las palabras de Lázaro (1999; p. 176) sobre la evaluación auténtica: «analiza e incluye a sujetos, familias, tareas, docentes y ambientes: todos están implicados en el proceso evaluador para recoger información y adoptar decisiones», pues ésta es la única forma para que las familias se impliquen en «su propio diagnóstico» y sientan que «les pertenece». En ese proceso el rol del experto en diagnóstico será el de «facilitador, colaborador, y recurso de aprendizaje» (Patton, 2002; p. 185), que encuentra pleno sentido en el marco de la evaluación auténtica («authentic assessment»).

Esto significa, como sugiere Gràcia (2003) al abordar la intervención, que para conseguir la plena participación de las familias no basta con considerar a las familias como ayudantes en el proceso. Simplemente, se trataría de un primer paso hacia la participación. El último paso consistiría en que las familias se convirtieran en las protagonistas de la intervención, lo que supondría su autogestión. A pesar de que éste es el objetivo final deseable para la autora, se debe reconocer que, al menos en un principio, este reto también se acompaña de una buena carga de angustia e incertidumbre para las familias. Por esta razón planteo un paso intermedio: La familia como miembro del equipo, advirtiendo que esto implica un cambio de concepción educativa que va desde la Educación Familiar, a la construcción cooperativa y democrática del diagnóstico y de sus posibles alternativas de intervención (Partnership Education). En palabras de Patton (2002; p. 185): «los participantes son co-iguales». El diagnóstico participativo nos remite a las necesarias competencias diagnósticas participativas y sociales, que se aproximan a lo que Moustakas (1995), según recoge Patton (2002), denominaría el desarrollo de una relación con las familias, expresada en el being-in (escuchar, aceptar, comprender...), being-for (tomar partido por el desarrollo), y being-with (escuchar, implicarse y ofrecer la propia perspectiva).

\subsection{Niveles de Diagnóstico Familiar}

El diagnóstico del contexto familiar, puede situarse en distintos niveles dependiendo, principalmente de si el análisis de la familia forma parte de un diagnóstico de naturaleza holística que incluye a la familia, entre otros factores (por ejemplo, los profesores, los programas educativos, las políticas educativas y sociales, los recursos de la escuela y del barrio...); o bien, se dirige al estudio de la familia como fenómeno educativo propio. Es decir, se trataría del diagnóstico de la familia como contexto, y/o el de la familia en sus contextos, siguiendo la aplicación del modelo ecológico de Bronfenbrenner. Así, a la hora de considerar las distintas dimensiones del contexto familiar, Freixa (2003) identifica los siguientes sistemas: el sistema individual (cada miembro de la familia), el sistema familiar (relaciones entre los miembros de la familia y su funcionamiento), y el sistema social (conformado por el contexto socioeconómico-cultural, y las relaciones entre los diferentes contextos).

De acuerdo con esta consideración sistémica, se entiende la familia como un complejo entramado de relaciones en el que los meca- 
nismos de influencia operan de dentro (del sistema personal y social) a fuera (sistema social), y viceversa, de fuera a dentro, concediendo la misma importancia relativa al papel de unos y otros miembros del sistema familiar. Por ejemplo, tanto influyen los hijos sobre los padres como los padres sobre los hijos; y al mismo tiempo estas influencias se encuentran en dinámico cambio en relación con el resto de los sistemas, y a su vez insertados en la cultura y en la historia. Se superan de este modo antiguas concepciones reduccionistas de tipo unidireccional (padres-hijos) y bidireccional (padres-hijos e hijos-padres), que además minimizaban el peso de factores como la familia extensa o el contexto social... entendiéndose todas las relaciones como transaccionales.

A pesar de su complejidad, el diagnóstico del contexto familiar está llamado a ser cada vez más demandado, dado el aumento constatado, sobre todo en las últimas décadas, en el auge de la orientación educativa familiar (Martínez González y Pérez Herrero, 2004; Martínez y Álvarez, 2002) ${ }^{[\mathrm{vi}]}$, y, por consiguiente, la evidente necesidad del diagnóstico para fundamentar las acciones orientadoras y de intervención socioeducativa. Se darían, pues, las condiciones para atreverme a afirmar que la familia se está convirtiendo cada vez más en un centro de interés diagnóstico, y en general social.

Desde el punto de vista de «los destinatarios» ${ }^{\text {[vii] }}$, podrían mencionarse cuatro niveles básicos de diagnóstico: para familias individuales, para familias con necesidades parecidas, para comunidades, y para el asesoramiento de personas con responsabilidades institucionales o políticas. El diagnóstico de las familias individuales nos remite a la «unicidad» de las necesidades familiares (Bradley-Johnson, 1994), y en general humanas, y se conecta con las funciones de consulta y consejo individualizado en la labor orientadora (Santana, 2003).
Desde planteamientos colectivos, hay otras posibilidades diagnósticas sobre grupos que a priori pueden presentar necesidades parecidas, de acuerdo con algunas características comunes (por ejemplo, familias con miembros discapacitados, familias con miembros toxicómanos, familias adoptivas, familias acogentes, familias con hijos recién nacidos, familias con hijos adolescentes, familias emigrantes, familias multiculturales...).

La determinación de esas necesidades comunes puede resultar de gran utilidad a la hora de confeccionar alternativas para la mejora de esas familias, que pueden surgir de la construcción participativa entre familias y «expertos en diagnóstico», así como con otros implicados en la situación. En estos casos adquieren sentido fórmulas de intervención como los programas de carácter experiencial, que vehiculan la reconstrucción compartida entre los padres de las actividades cotidianas (Maiquez, Rodrigo, Capote y Vermaes, 2000), cuyo fin último es hacer explícitos los sentimientos y las conductas, ampliando los repertorios de opciones alternativas y movilizando hacia el cambio a través de la reflexión conjunta.

De cualquier forma, y ya que el catálogo de diversidad en las familias es muy amplio, parece conveniente clasificarla en categorías más molares. De acuerdo con Rapoport y Rapoport (1982), existen las siguientes categorías de diversidad familiar: 1. Organizacional (respecto al trabajo familiar y externo); 2. Cultural (de nivel cultural, etnia, política, religión.... $)^{[\text {viii] }}$; 3. Clase social; 4. Cohorte (generaciones marcadas por las circunstancias temporales como, por ejemplo, un conflicto bélico); y 5. Ciclo vital que atraviesa la familia (Freixa, 1993; 2003). De acuerdo con este abanico de opciones de diversidad, se podrían ampliar las posibilidades anteriormente expuestas. Al mismo tiempo, se enlazarían con el diagnóstico de carácter comunitario, que según Ausín, López y Martín Izard (1998), se suele acompañar de funciones más bien correctivas, que general- 
mente se llevan a cabo con un soporte institucional (por ejemplo, las acciones municipales con colectivos específicos), aunque el objetivo deseable es llevar a cabo acciones preventivas.

De la misma forma, el diagnóstico sobre las familias puede tener como destinatario $a$ personas con responsabilidad política, que desean alcanzar un conocimiento profundo de la situación de las familias con vistas a implementar futuras acciones políticas, que efectivamente pueden plasmarse en dichas acciones comunitarias.

\subsection{Dimensiones de análisis en el con- texto familiar}

La complejidad y la amplitud de las dimensiones a considerar en el análisis del sistema familiar es tal que desbordaría cualquier ambición investigadora. Sin embargo, y sin ánimo de conseguir tal logro, dada la ausencia de un acuerdo unánime al respecto (Iglesias, 2005) me atreveré a sugerir aquellas dimensiones familiares que estimo más relevantes para el Diagnóstico en Educación, y que de alguna forma, vienen a recoger el abanico de opciones de diversidad en la familia. En primer lugar, señalaré las dimensiones físicas, sociodemográficas y organizacionales de la familia (INCE, 1998; Freixa, 2003; Moreno, 2003); en segundo lugar, las dimensiones cognitivo-educativas que englobarían creencias, metas, prácticas educa- tivas... (Triana y Rodrigo, 1993; Grusec y Goodnow, 1994; Freixa, 2003); finalmente, las dimensiones procesuales, que abarcarían los patrones de comunicación e interacción y las competencias requeridas para ello (Beavers y Hampson, 1995; Shapiro, 2001).

Todas estas dimensiones tienen cabida en el marco de la evaluación auténtica; que implica un abordaje holístico que reconoce tanto elementos contextuales internos y externos a la familia y sus interrelaciones, como aspectos cognitivos que median las acciones de los distintos miembros familiares. Sin embargo, revisten particular interés aquellos elementos procesuales susceptibles de captar de modo dinámico «la vida real» de las familias en su contexto natural. De cualquier modo, las dimensiones pueden ser objeto de análisis desde diversos procedimientos metodológicos, lo que puede condicionar que la información recogida encuentre significado desde la perspectiva de las propias familias, o se quede en un diagnóstico de «experto» alejado de sus protagonistas.

A continuación relacionaré los elementos de análisis que han sido considerados más sobresalientes para cada dimensión.

Los elementos de las dimensiones físicas, sociodemográficas y organizacionales los presentaré en la tabla 1 para facilitar su comprensión.

Tabla $\mathrm{n}^{\circ}$ 1: dimensiones de análisis del contexto familiar físicas, sociodemográficas y organizacionales

\begin{tabular}{|l|l|}
\hline DIMENSION FÍSICA, SOCIODEMOGRÁFICA, Y ORGANIZACIONAL \\
\hline Física & $\begin{array}{l}\text { Características de la vivienda (tamaño, estado, seguridad, higiene, orden...) } \\
\text { Características del barrio (servicios, equipamientos, seguridad...) } \\
\text { Canales de información (tipo, frecuencia, utilización...) } \\
\text { Recursos educativos (espacios, materiales, disponibilidad...) }\end{array}$ \\
\hline Sociodemográfica & $\begin{array}{l}\text { Situación económica de la familia (ingresos, gastos, procedencia, administración...) } \\
\text { Nivel cultural de la familia (estudios, profesión, intereses lúdicos, actividades culturales...) } \\
\text { Composición de la familia (número de personas convivientes, edades, parentesco, datos de } \\
\text { familia extensa...) } \\
\text { Datos de salud (con relevancia para el funcionamiento de la familia) } \\
\text { Características sociodemográficas del barrio }\end{array}$ \\
\hline Organizacional & $\begin{array}{l}\text { Organización y variedad de actividades cotidianas (días laborales y festivos) } \\
\text { Distribución de roles, tareas y funciones en los distintos miembros de la familia } \\
\text { Relaciones con otros contextos familiares y sociales (familia extensa, amigos, escuela, } \\
\text { asociaciones...) }\end{array}$ \\
\hline
\end{tabular}


El segundo grupo de elementos se corresponde con los elementos que Martínez González (1996) denomina actitudinales, y que tienen que ver con las cogniciones educativas de los miembros de la familia. Generalmente esta dimensión se ha aplicado más a los miembros adultos, por las dificultades de exploración cognitiva en los niños más pequeños. Con los adultos se han estudiado fundamentalmente las siguientes (Ceballos, 2004; Ceballos y Rodrigo, 1998):

- Creencias educativas: las ideas sobre el desarrollo y la educación de los hijos, también estudiadas como teorías implícitas por su carácter generalmente no explícito.

- Metas educativas: Los logros educativos que se pretenden conseguir, y que deberían guardar coherencia con las acciones educativas puestas en marcha. Muy cercanas se encuentran las expectativas, o el pronóstico que se realiza sobre las posibilidades de éxito o fracaso en la vida de un miembro de la familia.

- Prácticas educativas: las estrategias educativas puestas en marcha para alcanzar las metas.

- Ideologías y valores educativo-culturales de la familia.

La perspectiva de los niños más pequeños ha sido mucho menos estudiada (Mills, Nazar y Farell, 2002), y los elementos más analizados han sido (Ceballos y Rodrigo, 1998; Rodrigo, Janssens y Ceballos, 1999; Rodrigo, Janssens y Ceballos, 2001):

- La percepción y aceptación de los mensajes educativos de los adultos (generalmente los padres)

- Las atribuciones positivas o negativas que los niños realizan sobre las acciones de los adultos.

En las dimensiones procesuales se pueden resaltar:

- El nivel de comunicación y afectividad entre los distintos miembros de la familia

- El estilo relacional y el clima afectivo intra-familiar y extra-familiar
- Las estrategias de resolución de conflictos

- Las competencias emocionales, comunicativas y sociales en los distintos miembros de la familia.

Esta relación de dimensiones no ha pretendido ser exhaustiva, aunque sí ilustrativa de la variedad de aspectos a tener en cuenta en el diagnóstico familiar. Aunque la selección de la dimensiones a estudiar debe ser determinada en función del problema de investigación planteado, la amplitud de elementos de análisis presentada nos remite tanto a la diversidad de las familias como a la complejidad de su diagnóstico, lo que nos conduce también a la necesidad de fórmulas que permitan un análisis global de las realidades familiares.

\section{Métodos, técnicas e instrumentos}

Para apresar la rica variedad de dimensiones que acabo de citar, consideradas en su complejidad sistémica, parece razonable recurrir tanto a la metodología cuantitativa como a la cualitativa. Pues una visión multidisciplinar ayudará a vislumbrar la complejidad de los fenómenos que se pretenden aprehender, y a dar luz sobre las «cegueras paradigmáticas» (Morin, 2001). No obstante, cada profesional habrá de decidir en función de las finalidades del diagnóstico, y de las características de la situación, cuál es la metodología más adecuada al caso, y qué técnica o instrumentos deben acompañarla, mostrando la oportuna sensibilidad a los contextos (Patton, 2002). De cualquier forma y a pesar de las corrientes integradoras que preconizo en este artículo, persisten los partidarios exclusivos de una u otra opción metodológica.

Así, Cook (2003; p. 347) destaca la pertinencia de la metodología cuantitativa para tratar la complejidad del entramado familiar, pues «la mejor forma de tratar la complejidad es comenzar con pasos sencillos». De acuerdo con esta premisa las interacciones 
familiares intentan traducirse a sistemas de variables que describen relaciones causales, cuya incidencia se debe considerar con respecto al resto de sistemas ecológicos interrelacionados. De esta forma, se parte de modelos de interdependencia que pueden ser simples (por ejemplo, bidireccionales) o complejos, como el modelo SRM (Social Relations Model) de Kenny y La Voie (1984), y que nos sirven para describir y explicar diversas cuestiones familiares, facilitando la elaboración de teorías.

Sin embargo, desde planteamientos cualitativos, se sugiere que, a pesar de lo mucho que se ha investigado con la metodología cuantitativa y del gran número de resultados obtenidos, no se ha avanzado de igual modo en la comprensión de los pensamientos de los miembros de la familia (los cuales median sus interacciones), y como se ha expuesto en el apartado anterior, aún menos desde la perspectiva infantil. Por esta razón, Kuczynski y Dali (2003) consideran que la metodología cualitativa es la más apropiada para acceder al significado de todos los procesos de relación familiar ${ }^{[i x]}$.

En este sentido, los estudios etnográficos, usando una combinación de observaciones participantes y entrevistas, pueden resultar de gran utilidad para, por ejemplo, estudiar las relaciones de poder en la familia, o cómo se manifiestan éstas en las diferentes culturas. O también para investigar con los niños, o realizar estudios sobre familia desde un enfoque feminista, lo cual conecta directamente con la perspectiva crítica y «empoderadora» en la investigación y el diagnóstico de la familia. También debemos decir que desde estas corrientes parece existir un gran interés en la Teoría Fundamentada, que permitiría generar teorías a partir de los datos cualitativos; lo que resultaría particularmente conveniente cuando no exista una teoría bien desarrollada acerca del fenómeno familiar de interés. De todos modos, Greenstein (2001) puntualiza que ni toda la investigación cuantitativa tiene por qué ser positivista, ni toda investigación de corte cualitativo tiene por qué seguir la perspectiva de la Teoría Fundamentada.

En cuanto a las técnicas e instrumentos más empleados para el análisis de la familia, Grotevant y Carlson (1989) destacan los siguientes:

- Observaciones para analizar la interacción familiar, que se trasladan al registro de las distintas categorías observacionales y a la valoración en escalas de estimación (Rating-scales).

- Cuestionarios aplicados a los distintos miembros de la familia (sobre el funcionamiento y las relaciones de la familia, el estrés y su afrontamiento...).

Los cuestionarios y las escalas son los instrumentos más utilizados hasta el momento. La mayor parte de ellos provienen del ámbito anglosajón, lo que conlleva los correspondientes problemas de adaptación para poder ser empleados con nuestra población (Bayot, Hernández y de Julián, 2005). Algunos ejemplos típicos serían: para el diagnóstico de las dimensiones físicas, sociodemográficas y organizacionales, la Escala HOME (Home Observation for Measurement of the Environment) de Caldwell y Bradley; para la dimensión cognitivo-educativa, el CRPR (Child Rearing Practices Report) de Block; para la dimensión procesual, la Escala de Interacción de Competencia Familiar de Beavers.

Grotevant y Carlson (1989) también añaden otras técnicas que consideran de menor importancia desde su perspectiva, básicamente psicométrica: las técnicas proyectivas, las tareas experimentales, las entrevistas estructuradas y los autoinformes. Greenstein (2001) añade los métodos de encuesta, que pueden realizarse a través de cuestionarios enviados por correo postal, entrevistas telefónicas o personales. Además las TIC ofrecen nuevas posibilidades que permiten el acceso a un número indeterminado de perso- 
nas a través de los cuestionarios difundidos por Internet.

Sin embargo, este último autor apunta las limitaciones inherentes a los acercamientos estructurados y sugiere la idoneidad de la metodología cualitativa, si lo que se desea es captar los procesos que subyacen a las realidades familiares, lo que de nuevo hay que resaltar cuando el diagnóstico de la familia se orienta hacia la evaluación auténtica. En este sentido, se subraya la importancia de un diagnóstico que se realice en su contexto natural, para lo cual este último autor recomienda las siguientes estrategias:

- Estudios de casos, en los que se pueden usar diversas técnicas como las entrevistas semiestructuradas, o las observaciones participantes.

- Etnografías, especialmente aptas para el análisis de las distintas «culturas familiares», en las que la observación participante es la técnica más empleada.

- Grupos de discusión, tanto para obtener datos directamente, como para ayudar a desarrollar instrumentos más estructurados (por ejemplo, las encuestas).

Otra técnica interesante es el familiograma o genograma familiar, que facilita una visión rápida de la composición e historia de una familia, y los ecomapas que describen las relaciones que se establecen entre los diferentes subsistemas familiares, y los sistemas externos. Las historias de vida también pueden resultar un interesante complemento que contiene información altamente significativa para los distintos miembros de la familia, así como el análisis de biografías y documentos personales.

Sin duda, estos últimos grupos de técnicas contribuyen a un acercamiento contextualizado de las interacciones y significados familiares desde el punto de vista de cada uno de sus miembros, lo que ofrece la posibilidad de comparar y comprender sus distintas visiones. No obstante, ninguna de las técnicas e instrumentos mencionados es desdeñable, pues todos ellos, incluso los tests (Barootchi y Keshavarz, 2002) pueden encontrar cobijo desde la evaluación auténtica, respetando su carácter abierto, comprensivo y holístico.

\section{Limitaciones y posibilidades en el diagnóstico de la familia}

A pesar de todo lo expuesto hay que admitir que aún queda mucho por avanzar en el diagnóstico de la familia, lo cual puede considerarse comprensible dada la relativa juventud de este centro de interés para el Diagnóstico en Educación. Sin embargo, otras importantes limitaciones provienen de las dificultades propias de la metodología de investigación familiar, como las derivadas de la complejidad intrínseca de los fenómenos estudiados. No obstante, Martínez González (1996) puntualiza que en ocasiones se insiste demasiado en la complejidad del estudio de la familia, sin reparar en que estas dificultades son básicamente comunes a todos los objetos de estudio en las Ciencias Sociales. Sin duda, estos obstáculos nos conducen a la necesaria interdisciplinariedad, multiprofesionalidad y multimetodología.

A continuación daré cuenta de algunos de los aspectos señalados como elementos de dificultad en el estudio de la familia:

- La propia cercanía a nuestro modelo de familia, y a nuestro modelo de «normalidad familiar» que puede entorpecer una visión crítica al analizar «otras» familias $^{[\mathrm{x}]}$.

- Relacionado con el punto anterior está la enorme carga de valores que socialmente recibimos respecto a la familia, que hace que «todo el mundo» parezca «experto en temas familiares» (Martínez González, 1996).

- Consecuentemente, los problemas para evitar la influencia de la cultura en las técnicas y escalas de medida.

- La invisibilidad y privacidad de las relaciones familiares, lo cual pone de relieve la importancia de que el diagnóstico del contexto familiar sea un proceso participa- 
tivo, al tiempo que enfatiza las imprescindibles competencias sociales y participativas del experto en diagnóstico.

- La especificidad de las situaciones familiares, lo que nos remite al concepto de diversidad de las familias, $\mathrm{y}$ al análisis de sus distintas necesidades.

A pesar de este rosario de dificultades, y de que el diagnóstico del contexto familiar apenas esté despuntando en el Diagnóstico en Educación, creo que sus ventajas superan ampliamente a sus limitaciones. Pues si se prescinde del análisis del contexto familiar se renuncia, en definitiva, a la comprensión holística de la realidad educativa. Acceder a las claves de la familia ayudará muy posiblemente a comprender mejor la mayoría de las diversas situaciones educativas: las dificultades de aprendizaje, los problemas de la integración multicultural, la marginación social, la discriminación a causa del género, la falta de competencias sociales y emocionales... todas estas cuestiones pueden encontrar respuestas más claras a través del diagnóstico del contexto familiar. Respuestas que nos dirijan hacia la dimensión diagnóstica transformadora y emancipadora. Todo ello en coherencia con el compromiso social adquirido por el Diagnóstico en Educación, y en consonancia con las expectativas abiertas por la posibilidad de acciones globales de carácter institucional en «partenariado».

\section{Referencias bibliográficas}

Alberdi, I. (1999). La nueva familia española. Madrid: Taurus.

Álvarez González, B. (2003). Orientación Familiar. Intervención Familiar en el ámbito de la diversidad. Madrid: Sanz y Torres.

Ausín, T., López, D. y Martín-Izard, J. F. (1998). La intervención familiar. En R. Bisquerra (coord.), Modelos de orientación e intervención psicopedagógica (pp. 391403).

Barootchi, N. y Keshavartz, M. H. (2002). Assessment of achievement through portfolios and teacher made tests. Educational Research 44 (3), 279-288.
Bartolomé, M., Cabrera, F., Espín, J., Marín, M. A., J., \& Rodríguez, M. (2000). El enfoque intercultural en educación e inserción sociolaboral: algunas líneas de investigación desde la realidad catalana. Qurriculum, 14, 41-76.

Baumrind, D. (1973). The development of instrumental competence through socialization. En A. D. Pick (ed.) Minnesota Symposia on Child Psychology (7, 3-46). Minneapolis: University of Minnesota Press.

Bayot, A., Hernández, J.V. y de Julián, L.F. (2005). Análisis factorial exploratorio y propiedades psicométricas de la escala de competencia parental percibida. Versión para padres/madres (ECPP-p). Revista Electrónica de Investigación y EValuación Educativa, 11 ,

http://www.uv.es/RELIEVE/v11n2/RELIE

VEv11n2_2.htm . Consultado el 6-10-2005.

Beavers, W. R. \& Hampson, R. B. (1995).

Familias exitosas. Barcelona: Paidós.

Bernardes, J. (1997). Family studies. Londres: Routledge.

Bradley-Johnson, S. (1994). Psychoeducational assessment of students who are visually impaired or blind. Austin (Texas): Pro.ed

Cabrera, F., Espín, J., Marín, M. A. \& Rodríguez, M. (1998). Diagnóstico del contexto de las familias inmigrantes. En M. Bartolomé, Diagnóstico a la escuela multicultural (pp. 127-159). Barcelona: Cedes.

Ceballos, E. (2004). La legibilidad de las prácticas y metas educativas de las madres en relación con su contenido y organización. Universidad de la laguna: Servicio de Publicaciones.

Ceballos, E. \& Rodrigo, M. J. (1998). Las metas y estrategias de socialización entre padres e hijos. En M. J. Rodrigo y J. Palacios (coords.) Familia y Desarrollo Humano (pp 225-244). Madrid: Síntesis.

Cook, W. L. (2003). Quantitative methods for deductive (theory-testing) research on parent-child dynamics. In L. Kuczynski (ed.), Handbook of Dynamics in parentchild relations (pp. 347-372). Thousand Oaks CA: Sage. 
Cusinato, M. (2002). Research on family resources and needs across the world. Milán: LED

Esteve, J. M. (2003). La tercera revolución educativa. Barcelona: Paidós.

Eurostat (1998). Portrait social de l'Europe: septembre 1998. Luxembourg: Office des Publications Officielles des Communautés Européennes.

Freixa, M. (1993). El ciclo vital de la familia de la persona con disminución psíquica. Siglo Cero 146, 45-55.

Freixa, M. (2003). Diagnóstico del contexto familiar. En M. Álvarez y R. Bisquerra (coords.) Manual de Orientación y Tutoría (pp. 464/79-93). Barcelona: Praxis.

Giddens, A. (1999). Reith Lectures. Runaway world. London: BBC.

Gràcia, M. (2003). Comunicación y lenguaje en primeras edades: intervención con familias. Lleida: Milenio.

Greenstein, T. N. (2001). Methods of Family

Research. Thousand Oaks CA: Sage.

Grotevant, H. D. \& Carlson, C. I. (1989). Family assessment. A guide to methods and measures. Nueva York: The Guilford Press.

Grusec, J. E. \& Goodnow, J. J. (1994). Impact of parental discipline methods on child's internalization of values: A reconceptualization of current points of view. Developmental Psychology, 30, 4-19.

Hernández Blasi, C. (1996). Bronfenbrenner y los modelos ecológicos. En R. Clemente y C. Hernández Blasi, Contextos de desarrollo psicológico y educación (pp. 91-111). Málaga: Aljibe.

Iglesias, M.J. (2005). Diagnóstico escolar. Teorías, ámbitos y técnicas. Madrid: Pearson Prentice Hall.

INCE (Instituto Nacional de Calidad y evaluación) (1998). Familia y escuela. Diagnóstico del Sistema Educativo. La escuela secundaria obligatoria. Madrid: MEC.

Kenny, D. A. \& La Voie, L. (1984). The social relations model. In L. Berkowitz (Ed.), Advances in Experimental Social Psychology (Vol. 18, pp. 141-182). Nueva York:
Kuczynski, L. \& Dali, K. (2003). Qualitative methods for inductive (theory-generating) research. In L. Kuczynski (ed.), Handbook of Dynamics in parent-child relations (pp. 373-392). Thousand Oaks CA: Sage.

Lázaro, A. (1999). Revisión de las tendencias en la evaluación psicopedagógica. Contextos educativos, 2, 167-180.

Maganto, J. M., Bartau, I. \& Etxeberria, J. (2003a). La participación de los hijos en el trabajo familiar. Revista de Investigación Educativa, 21 (1), 249-270.

Maganto, I., Bartau, I. \& Etxeberria, J. (2003b). La participación en el trabajo familiar: un reto educativo y social. Relieve, 9 (2).

http://www.uv.es/RELIEVE/v9n2/RELIEV Ev9n2_4.htm. Consultado el 20-10-2003.

Maiquez, M. L, Rodrigo, M. J., Capote, M. C., \& Vermaes, I. (2000). Enseñar y aprender en la vida cotidiana: un programa experiencial para padres. Madrid: Aprendizaje Visor.

Marín, M. A. y Rodríguez Espinar, S. (2001). Prospectiva del Diagnóstico y la Orientación. Revista de Investigación Educativa, 19 (2), 315-364.

Martínez González, R. A. (1996). Familia y Educación. Servicio de Publicaciones de la Universidad de Oviedo.

Martínez González, R. A. (1999). Orientación Educativa para la Vida Familiar. Revista Española de Orientación y Psicopedagogía, 10 (17), 115-127.

Martínez González, R. A. (2004). Familia y Escuela. Ponencia impartida en la II Edición del Master de Intervención Familiar de la Universidad de La Laguna. La Laguna, abril 2004

Martínez González, R. A. y Pérez Herrero, M. H. (2004). Evaluación e intervención educativa en el campo familiar. Revista Española de Orientación y Psicopedagogía 15, (1), 89-104.

Martínez, M. C. \& Álvarez, B. (2002). Orientación familiar. Madrid: UNED.

Mills, R. S. L., Nazar, J. y Farell, H. M. (2002). Child and parent perceptions of 
hurtful messages. Journal of Social and Personal Relationships 19 (6), 731-754.

Moreno, M. C. (2003). Material docente del Master de Intervención familiar.

Morín, E. (2001). Los siete saberes necesarios para la educación del futuro. Barcelona: Paidós.

Moustakas, C. (1995). Being- in, being-for, being-with. Northvale, NJ: Jason Aronson

Palacios, J. \& Rodrigo, M. J. (1998). La familia como contexto de desarrollo humano. En M. J. Rodrigo y J. Palacios (coords.) Familia y Desarrollo Humano (pp 25-44). Madrid: Síntesis.

Patton, M. Q. (2002). Qualitative research \& evaluation methods. Thousand Oaks CA: Sage.

Pérez Díaz, V., Chuliá, E. \& Valiente, C. (2000). La familia española en el año 2000. Madrid: Visor.

Rapoport, R. \& Rapoport, R. N. (1982). British families in transition. In R. N. Rapoport et al (eds.), Families in Britain (pp. 475499). Londres: Rouledge.

Rodrigo, M. J., Janssens, J.M.A.M. \& Ceballos, E. (1999). Do Children's Perceptions and Attributions Mediate the Effects of Mothers' Child-rearing Actions? Journal of Family Psychology, vol. 13, 4, 508-522.

Rodrigo, M. J., Janssens, J.M.A.M. \& Ceballos, E. (2001). Reasoning and action complexity: Sources and consequences on maternal child-rearing behavior. International Journal of Behavioral Development, Vol. 25, 1, 50-59.

Romero, F. (1998). Los centros de orientación familiar en España. Un análisis crítico. Servicio de Publicaciones de la Universidad de Las Palmas de Gran Canaria.

Santana, L. E. (1993). Los dilemas en la orientación educativa. Irala (Argentina): Cincel.

Santana, L. E. (2001). Orientación, estudios prospectivos y postmodernidad. En L. E. Santana (coord.) Trabajo, Educación y Cultura. Un enfoque interdisciplinar (pp. 123150). Madrid: Pirámide.
Santana, L. E. (2003). Orientación educativa e intervención psicopedagógica. Madrid: Pirámide.

Shapiro, L. E. (2001). La inteligencia emocional de los niños. Una guía para padres y maestros. Madrid: Suma de letras.

Simón, M. I., Correa, N., Rodrigo, M. J. y Rodríguez, M. A. (1998). Desarrollo y educación familiar en niños con cursos evolutivos diferentes. En M. J. Rodrigo y J. Palacios (coords.), Familia y desarrollo Humano (pp.445-464). Madrid: Alianza Editorial.

Triana, B. \& Rodrigo, M. J. (1993). Las teorías implícitas de los padres sobre la infancia y el desarrollo. En M. J. Rodrigo, A. Rodríguez y J. Marrero (comps.), Las teorías implícitas. Una aproximación al conocimiento cotidiano (pp. 203-242). Madrid: Visor.

\section{NOTAS}

[i] Las nuevas «guías familiares» son proporcionadas por los modelos idílicos e irreales que aparecen en la televisión.

[ii] También es llamativo el espectacular aumento del número de hogares unipersonales, que alcanzan el 13,3\% (frente al 26'9\% de la UE), según apuntan Martínez y Álvarez (2002).

[iii] Aún así, Maganto, Bartau y Etxeberria (2003a) destacan que a día de hoy prevalece una desequilibrada sobrecarga de roles familiares sobre los hombros de las mujeres, quienes realizan tres veces más tareas domésticas que los hombres, y llevan el 90\% de las responsabilidades acerca del cuidado de los niños: planean, organizan, delegan, supervisan y programan. Esta desigualdad se perpetúa en la desigual participación en el trabajo familiar de los hijos (Maganto, Bartau y Etxeberria, 2003b).

[iv] Esteve (2003) señala que este libro ha sido traducido a treinta y nueve idiomas, $\mathrm{y}$ que sus ventas han alcanzado más de treinta millones de ejemplares, siendo calificado como «el libro para padres más vendido del 
mundo». En dicho manual, de corte psicoanalítico, se invita a los padres a no coartar la expresión de los sentimientos de sus hijos, ni a frustrarles, permitiéndoles todo tipo de excesos.

[v] La escuela y la familia, entre otras, resultan instituciones formativas cuyos límites se presentan como difusos. (Marín y Rodríguez Espinar, 2001).

[vi] Romero (1998) señala que su presencia en España ha llegado con retraso respecto a Europa y EEUU, si bien es aún escasa; registra 32 centros de orientación familiar hasta 1998, la mayoría abiertos a partir de 1990, por lo que la previsión es de un gran aumento. [vii] La noción de destinatarios y agentes del diagnóstico se diluye en la concepción participativa del diagnóstico.

[viii] Especial interés reviste en este sentido el diagnóstico del contexto de las familias inmigrantes (Cabrera, Espín, Marín y Rodríguez, 1997; Bartolomé, Cabrera, Espín, Marín y Rodríguez, 2000)

[ix] Según sus propias palabras: «Qualitative methods attempt to study the lived experiences of parents and children in their relationships and the meanings that they construct of those experiences from their own perspective» (p. 375).

[x] En palabras de Bernardes (1997; p. 54): «is the study of family life possible, given that we are all so familiar with our own family lives?».

\section{$\underline{\text { ABOUT THE AUTHORS / SOBRE LOS AUTORES }}$}

Esperanza Ceballos (eceballo@ull.es). Profesora Titular del Área de Métodos de Investigación y Diagnóstico en Educación de la Universidad de La Laguna. Sus líneas de investigación se centran preferentemente en el diagnóstico familiar y la prevención del riesgo social en la infancia y adolescencia. 


\title{
ARTICLE RECORD / FICHA DEL ARTÍCULO
}

\begin{tabular}{|c|c|}
\hline $\begin{array}{l}\text { Reference / } \\
\text { Referencia }\end{array}$ & $\begin{array}{l}\text { Ceballos, Esperanza (2006). Dimensiones de análisis del Diagnóstico en Educación: El Diagnóstico del } \\
\text { Contexto Familiar. Revista ELectrónica de Investigación y EValuación Educativa, v. 12, n. 1. } \\
\text { http://www.uv.es/RELIEVE/v12n1/RELIEVEv12n1_4.htm. Consultado en (poner fecha). }\end{array}$ \\
\hline Title / Título & $\begin{array}{l}\text { Dimensiones de análisis del Diagnóstico en Educación: El Diagnóstico del Contexto Familiar [Analysis } \\
\text { dimensions in educational diagnosis: Diagnosis of Family Context] }\end{array}$ \\
\hline $\begin{array}{l}\text { Authors / } \\
\text { Autores }\end{array}$ & Esperanza Ceballos \\
\hline $\begin{array}{l}\text { Review / } \\
\text { Revista }\end{array}$ & Revista ELectrónica de Investigación y EValuación Educativa (RELIEVE), v. 12, n. 1 \\
\hline ISSN & $1134-4032$ \\
\hline $\begin{array}{l}\text { Publication } \\
\text { date / } \\
\text { Fecha de } \\
\text { publicación }\end{array}$ & $\begin{array}{l}2006 \text { (Reception Date: } 2005 \text { December 18; Approval Date: } 2006 \text { March 14; Publication Date: } 2006 \\
\text { March 14) }\end{array}$ \\
\hline $\begin{array}{l}\text { Abstract / } \\
\text { Resumen }\end{array}$ & $\begin{array}{l}\text { Diagnosis of family context has been a subject relatively little considered in the scope of educational } \\
\text { diagnosis. Even though whose relevance has been underestimated from the assessment classical perspec- } \\
\text { tive, an increasing interest is being aroused, especially on account of holistical and ecological trends. } \\
\text { This article intends to offer a synthesis of most relevant topics to be considered in diagnosis of family } \\
\text { context, emphasizing its importance for the approach to authentic evaluation. } \\
\text { El diagnóstico del contexto familiar ha sido un tema relativamente poco tratado en el ámbito del dia- } \\
\text { gnóstico en educación, y cuya relevancia ha sido soslayada desde la perspectiva clásica del assessment. } \\
\text { Sin embargo, se constata un interés creciente suscitado especialmente al hilo de las tendencias holísticas } \\
\text { y ecológicas en el diagnóstico. Este artículo pretende ofrecer una síntesis de los tópicos más relevantes a } \\
\text { tener en cuenta en el diagnóstico del contexto familiar, subrayando la importancia del análisis de este } \\
\text { contexto para la aproximación a una evaluación auténtica. }\end{array}$ \\
\hline $\begin{array}{l}\text { Keywords } \\
\text { Descriptores }\end{array}$ & $\begin{array}{l}\text { Diagnosis, family context, authentic evaluation, empowerment } \\
\text { Diagnóstico contexto familiar, evaluación auténtica, fortalecimiento }\end{array}$ \\
\hline \begin{tabular}{l|} 
Institution / \\
Institución
\end{tabular} & Universidad de La Laguna (España) \\
\hline $\begin{array}{l}\text { Publication } \\
\text { site / } \\
\text { Dirección }\end{array}$ & http://www.uv.es/RELIEVE \\
\hline $\begin{array}{l}\text { Language / } \\
\text { Idioma }\end{array}$ & Spanish (Title, abstract and keywords in english) \\
\hline
\end{tabular}

\section{Revista ELectrónica de Investigación y EValuación Educativa (RELIEVE)}

\author{
[ ISSN: 1134-4032 ] \\ (c) Copyright, RELIEVE. Reproduction and distribution of this articles it is authorized if the content is no modified \\ and their origin is indicated (RELIEVE Journal, volume, number and electronic address of the document). \\ (C) Copyright, RELIEVE. Se autoriza la reproducción y distribución de este artículo siempre que no se modifique el \\ contenido y se indique su origen (RELIEVE, volumen, número y dirección electrónica del documento).
}

